\title{
PRE-DESIGNING OF A MECHATRONIC SYSTEM USING AN ANALYTICAL APPROACH WITH DYMOLA
}

\author{
Ghazoi Hamza \\ Mechanics Modeling and Production Research Laboratory (LA2MP), National School of Engineers of Sfax (ENIS), Sfax, \\ Tunisia and \\ Laboratory of Engineering of the Mechanical Structures and Materials (LISMMA), High Institute of Mechanic of Paris \\ (SUPMECA), Saint-Ouen Cedex, France; e-mail: hamza.ghazoi@gmail.com \\ Jean-Yves Choley, Moncef Hammadi, Alain Riviere \\ Laboratory of Engineering of the Mechanical Structures and Materials (LISMMA), High Institute of Mechanic of Paris \\ (SUPMECA), Saint-Ouen Cedex, France
}

Maher Barkallah, Jamel Louati, Mohamed Haddar

Mechanics Modeling and Production Research Laboratory (LA2MP), National School of Engineers of Sfax (ENIS), Sfax, Tunisia

\begin{abstract}
This paper presents a pre-dimensioning method applied to a mechatronic system and regarding the vibrational aspect, through a simple modeling process in Dymola environment. We study the vibration transmission between dynamic exciters (motors) and receivers (electronic cards) which are located on a simply supported rectangular plate, using an analytical approach. This new method will allow us to perform representative and robust modeling and simulation. The solution for this issue would be a pre-sizing and pre-positioning procedure. It aims to determine a set of possible technical solutions and principal characteristics before the definitive choice of components and precise sizing of the system. The presented method predicts also behaviour of the mechatronic system. In order to validate the model with respect to the finite element method, selected simulation results are presented.
\end{abstract}

Keywords: pre-dimensioning, Dymola, analytical method, vibration, rectangular plate

\section{Introduction}

Mechatronics is the synergistic integration of a physical system, actuators, electronic components and sensors, etc., through the design process (Craig and Stolfi, 2002) and (Wang et al., 2013) in order to develop new products more efficient and more reliable. It is very common in our daily life as well as in industry. In addition, it affects many applications in various fields such as transportation, automotive systems and production (Elmqvist et al., 2001).

In order to minimize the cost as well as the time to market while improving their quality, it is important to take a great effort from the early phases of the design cycle of mechatronic systems. Thus, most important methodological efforts must be considered in the upstream phase of product development. The development process of a mechatronic system includes three phases which are the requirement analysis, pre-sizing and detailed sizing. The preliminary design is performed based on various feedback experiments and a set of specifications. Moreover, it should allow determining the system architecture and its setting (Liscouët et al., 2012). For this reason, it may be necessary to describe the main phenomena involved in the system such as vibrational and thermal behaviour. Accordingly, a mechatronic designer uses different modeling tools to elaborate multi-physics models in the multidisciplinary design process of mechatronic systems. 
In recent years, many researches, based on techniques such as multi-criteria decision making and poly-optimization, have been working on the integrated preliminary design and optimization of mechatronic systems. For instance, Hammadi et al. (2012b) proposed to aggregate several important criteria in one multi-criteria indicator to evaluate the mechatronic system performance in the preliminary design level. Moreover, Tarnowski et al. (2011) proposed the poly-optimization paradigm as an engineering design methodology for mechatronic system design. Every designer involved in the design process has to solve a design task as an inverse problem in an iterative way. The poly-optimization allows designers to make decision as a trade-off between conflicted criteria to find a sub-set of non-dominated solutions. Poly-optimization can be more interesting than aggregating many criteria in one multi-criteria indicator. However, a further development is required to automate the decision making based on poly-optimization.

To reduce the computing time during the system analysis and optimization of the mechatronic design, surrogate models can substitute the costly analysis models. A methodology based on the surrogate modeling technique combined with Modelica language was proposed by Hammadi et al. (2012a) for the optimization of electric vehicles. Although this method reduces the computing time during the optimization process, the elaboration of surrogate models is somewhat long and mechatronic designers have to verify the accuracy of the surrogate models before including them in the optimization process.

Long computing time in the analysis and optimization of mechatronic systems is due to the methods used to model and solve the governing equations of the mechatronic system and its components. In fact, the components modelled with partial differential equations (PDEs) are the major source of difficulties in the analysis and optimization of mechatronic systems. However, PDEs are strongly required to analyse the vibrating aspect of mechatronic systems. A vibrating component in mechatronic systems can be modelled with simple beams or plates. The governing equation of vibrating plate theories have been solved through three types of solutions: numerical methods, exact analytical methods and semi analytical methods.

In the case when the plate has all edges simply supported and a lateral force acts over the plate surface, Navier presented an exact method to obtain the solution of bending by using a double series Fourier approach, see Szilard (2004) and Ventsel and Krauthammer (2001). This method can be extended to an orthotropic plate subjected to dynamic excitation (Romanelli and Laura, 2001).

By using a single trigonometric series, Levy developed a method to solve the bending problem of a rectangular plate with two opposite sides simply supported and the remaining edges with arbitrary conditions (clamped, simply supported guided, etc.), see Ventsel and Krauthammer (2001).

In addition, Lagrange's multipliers approach was introduced by Vera et al. (2005) to study the problem of a plate supporting a 2-dof spring-mass system elastically mounted. However, it is not always possible to obtain analytical solutions, especially for complex structures. For this reason, many efforts have been dedicated to develop approximate methods, such as Galerkin's type approximate procedure. For instance, Laura and Duran (1975) used a simple polynomial approximation and Galerkin's method to determine the response of a thin rectangular plate clamped at four edges and subjected to uniformly distribute sinusoidal excitation. In this method, the approximate solution is expressed in terms of polynomials which satisfy all the prescribed boundary conditions. An untruncated infinite series superposition method (UISSM) was presented by Bhaskar and Sivaram (2008) for a rectangular plate with arbitrary boundary conditions and an arbitrary transverse load. The achieved solutions satisfy different requirements and they are obtained as a simple combination of analytically component solutions. The Rayleigh Ritz method is one of the most famous approximate methods; it is a useful approach for many eigenvalue problems (Leissa, 2005). This method belongs to the so-called variational methods; it assumes that the solution of the variational problem can be approximated by a suitable linear combina- 
tion of the coordinate function. This method can be used for both thin and thick rectangular plates with different combinations of boundary conditions.

Various numerical methods have been developed to deal with the vibration problem of rectangular plates with mixed and non-uniform boundary conditions. Each of them can be classified as either a local method (finite difference method, finite element method) or a global method (differential quadratic method, Ritz method). In general, global methods are more accurate than the local ones. By contrast, local methods are more flexible to handle complex geometries and boundary conditions. A discrete singular convolution algorithm, as a potential approach, was introduced by Wei et al. (2001) to study this kind of problems. The mathematical fundamentals of this algorithm are based on the theory of distributions (Schwartz, 1951). This approach has the advantages of both methods (local and global).

The natural frequencies and the mode shapes of a rectangular plate, carrying any number of translational springs and point masses are determined by using the analytical and numerical combined method (ANCM) (Wu and Luo, 1997). By obtaining the analytical closed form solutions for the mode shapes and natural frequencies, based on the mode superposition theory, the eigenvalue equation of the plate with these elements can be derived. This approach prevents the algebraic difficulties of the analytical method. Moreover, it consumes less computation time if one compares it with the finite element method (FEM).

A plate vibrating in a mechatronic system can cause problems to various components which are interconnected such as electronic devices which are essential and vital components in any mechatronic systems. Moreover, they must be able to operate over the important life cycle and survive environmental issues such as temperature and vibration (Veprik and Babtisky, 2000) and (Mchira and Pecht, 2002) to which they are sensitive. Consequently, for the preliminary design phase, the objective is to predict how the electronic component will implement completely and correctly the functions specified in the requirements within the constraints of the device, the environments the component will function within and the defined interfaces. During this phase, the designer should maintain a system perspective and see the interaction with the rest of the system in order to identify inconsistencies and ambiguities.

The main purpose of this work is to present a pre-dimensioning method based on an analytical approach and taking into consideration the effect of vibration. More precisely, we focus on the effect of dynamic disturbances produced by motors and transmitted to the electronic cards through the plate. In order to predict the behaviour of the mechatronic system, robust modeling and simulations with DYMOLA are carried out. We investigate the sensitivity of the system for several parameters and different architectures in order to develop an accurate and reliable design.

The organization of this paper is as follows: the first section deals with the introduction, in Section 2 we introduce the analytical formulation of the problem, in Section 3 we present the modeling of the system. Results of simulations are presented and discussed in Section 4 with comparison to FEM. The conclusion of our paper is given in Section 5.

\section{Theoretical background}

\subsection{Analytical formulation of plate vibration}

Before developing the analytical model, we need to make some hypothesis and simplifications. Indeed, the simply supported rectangular plate will be considered as homogeneous, linearly elastic, isotropic and uniformly thin. Neither the shear deformations nor in plane forces are taken into account. The relatively low frequencies induced by electric motors and the small thickness of the plate compared to the other dimensions allow us to consider only transverse vibration. The excitations in many mechatronic systems can be considered as a random process. 
However, for simplification reasons, we will deal only with deterministic structural parameters. Therefore, only a harmonic excitation is considered in this study.

The coordinate system of the plate is presented in Fig. 1. In these conditions, the classical plate governing differential equation of motion can be written as

$$
D \nabla^{4} w=-\rho h \frac{\partial^{2} w}{\partial t^{2}}+f(x, y, t)
$$

where

$$
f(x, y, t)=\sum_{i=1}^{N} f_{i}
$$

$\nabla^{2}$ is the Laplacian operator, $w(x, y, t)$ is the transverse deflection, $N$ is the number of electric motors. $D, \rho, h$ and $f(x, y, t)$ present respectively the flexural rigidity, density of the plate material, plate thickness and the transverse external loadings

$$
D=\frac{E h^{3}}{12\left(1-v^{2}\right)}
$$

where $E$ and $v$ are respectively Young's modulus and Poisson's ratio.

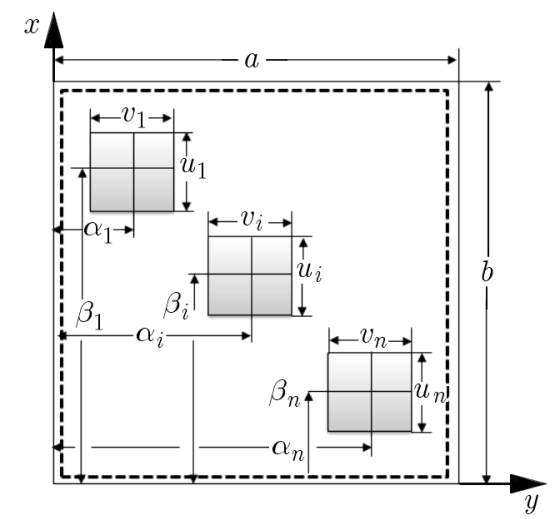

Fig. 1. Spatial distribution of dynamic pressures on the plate (top view)

We consider that every motor mounted on the plate induces a dynamic excitation over a finite area. In this case, we consider that a uniformly distributed $p \cos (\omega t)$ type force, equivalent to the excitation generated by the motor, acts over a rectangular portion of the plate of dimensions $u_{i}$ and $v_{i}$, where the coordinates of the centroid are $\left(\alpha_{i}, \beta_{i}\right)$

$$
f_{i}=p_{i} \cos \left(\omega_{i} t\right)
$$

where, $p_{i}$ is the intensity of the distributed load, $\omega_{i}$ is the angular frequency of the dynamic excitation. Then

$$
f(x, y, t)=\sum_{i=1}^{N} p_{i} \cos \left(\omega_{i} t\right)
$$

The boundary conditions for a simply supported plate are the following

$$
w=\left.0\right|_{x=0, a} \quad \frac{\partial^{2} w}{\partial x^{2}}=\left.0\right|_{x=0, a} \quad w=\left.0\right|_{y=0, b} \quad \frac{\partial^{2} w}{\partial y^{2}}=\left.0\right|_{y=0, b}
$$

In the following, we use Navier's principle to establish the dynamic equation of the structure. 
The spatial distribution of the dynamical loading has been expanded into the double Fourier series (Szilard, 2004)

$$
f_{i}(x, y, t)=\cos \left(\omega_{i} t\right) \sum_{n=1}^{\infty} \sum_{m=1}^{\infty} b_{n m}^{i} \sin \frac{n \pi x}{a} \sin \frac{m \pi y}{b}
$$

The coefficients of the Fourier series can be calculated as

$$
\begin{aligned}
& b_{n m}^{i}=\frac{4 p_{i}}{a b} \int_{\alpha_{i}-u_{i} / 2}^{\alpha_{i}+u_{i} / 2} \int_{\beta_{i}-v_{i} / 2}^{\beta_{i}+v_{i} / 2} \sin \frac{n \pi x}{a} \sin \frac{m \pi y}{b}=\frac{16 p_{i}}{\pi^{2}} B_{n m}^{i} \\
& B_{n m}^{i}=\frac{1}{n m} \sin \frac{n \pi \alpha_{i}}{a} \sin \frac{n \pi u_{i}}{2 a} \sin \frac{m \pi \beta_{i}}{b} \sin \frac{m \pi v_{i}}{2 b}
\end{aligned}
$$

Following this approach, based on the superposition method, the expression of the deflected surface $W(x, y, t)$ can be written as in the following

$$
w(x, y, t)=\sum_{i=1}^{N} w_{i} \cos \left(\omega_{i} t\right)=\sum_{i=1}^{N} \cos \left(\omega_{i} t\right) \sum_{n=1}^{\infty} \sum_{m=1}^{\infty} C_{n m}^{i} \sin \frac{n \pi x}{a} \sin \frac{m \pi y}{b}
$$

then

$$
\begin{gathered}
w(x, y, t)=\cos \left(\omega_{1} t\right) \sum_{n=1}^{\infty} \sum_{m=1}^{\infty} C_{n m}^{1} \sin \frac{n \pi x}{a} \sin \frac{m \pi y}{b}+\cos \left(\omega_{2} t\right) \sum_{n=1}^{\infty} \sum_{m=1}^{\infty} C_{n m}^{2} \sin \frac{n \pi x}{a} \sin \frac{m \pi y}{b} \\
+\ldots+\cos \left(\omega_{N} t\right) \sum_{n=1}^{\infty} \sum_{m=1}^{\infty} C_{n m}^{N} \sin \frac{n \pi x}{a} \sin \frac{m \pi y}{b}
\end{gathered}
$$

with

$$
\begin{aligned}
& C_{n m}^{1}=\frac{16 p_{1} B_{n m}^{1}}{\pi^{2}\left\{D\left[\left(\frac{\pi n}{a}\right)^{2}+\left(\frac{\pi m}{b}\right)^{2}\right]^{2}-\rho h \omega_{1}^{2}\right\}} \quad C_{n m}^{2}=\frac{16 p_{2} B_{n m}^{2}}{\pi^{2}\left\{D\left[\left(\frac{\pi n}{a}\right)^{2}+\left(\frac{\pi m}{b}\right)^{2}\right]^{2}-\rho h \omega_{2}^{2}\right\}} \\
& \vdots \\
& C_{n m}^{N}=\frac{16 p_{N} B_{n m}^{N}}{\pi^{2}\left\{D\left[\left(\frac{\pi n}{a}\right)^{2}+\left(\frac{\pi m}{b}\right)^{2}\right]^{2}-\rho h \omega_{N}^{2}\right\}}
\end{aligned}
$$

Expression of deflection (2.12) automatically satisfies the prescribed boundary conditions.

For a simply supported plate, the natural frequencies are

$$
\omega_{n m}=\pi^{2}\left[\left(\frac{m}{a}\right)^{2}+\left(\frac{n}{b}\right)^{2}\right] \sqrt{\frac{D}{\rho h}}
$$

For the case of one installed motor, the amplitudes of the plate bending moments are

$$
M_{x}=D\left(\frac{\partial^{2} w}{\partial x^{2}}+\nu \frac{\partial^{2} w}{\partial y^{2}}\right) \quad M_{y}=D\left(\nu \frac{\partial^{2} w}{\partial x^{2}}+\frac{\partial^{2} w}{\partial y^{2}}\right)
$$

then

$$
\begin{aligned}
& M_{x}=\frac{16 p_{1} a^{2}}{\pi^{2}} \sum_{n=1}^{\infty} \sum_{m=1}^{\infty} \frac{B_{n m}}{F_{n m}-R^{2} F_{11}}\left[n^{2}+v m^{2}\left(\frac{a}{b}\right)^{2}\right] \sin \frac{n \pi x}{a} \sin \frac{m \pi y}{b} \\
& M_{y}=\frac{16 p_{1} a^{2}}{\pi^{4}} \sum_{n=1}^{\infty} \sum_{m=1}^{\infty} \frac{B_{n m}}{F_{m n}-R^{2} F_{11}}\left[v n^{2}+\left(\frac{m a}{b}\right)^{2}\right] \sin \frac{n \pi x}{a} \sin \frac{m \pi y}{b}
\end{aligned}
$$


with

$$
F_{n m}=\left[n^{2}+m^{2}\left(\frac{a}{b}\right)^{2}\right]^{2} \quad R=\frac{\omega}{\omega_{11}}
$$

The kinetic energy is

$$
T=\frac{1}{2} \rho h \int_{S}\left(\frac{\partial w}{\partial t}\right)^{2} d x d y
$$

In order to simplify the problem, we take $m=n=1$ with $N=1$. Then

$$
\begin{aligned}
& w=\cos \left(\omega_{1} t\right) C_{11}^{1} \sin \frac{\pi x}{a} \sin \frac{\pi y}{b} \\
& T=\frac{1}{2} \rho h \int_{0}^{a} \int_{0}^{b}\left(-\omega_{1} \sin \left(\omega_{1} t\right) C_{11}^{1} \sin \frac{\pi x}{a} \sin \frac{\pi y}{b}\right)^{2} d x d y
\end{aligned}
$$

so

$$
T=\frac{1}{8} \rho h\left[-\omega_{1} \sin \left(\omega_{1} t\right) C_{11}^{1}\right]^{2} a b
$$

The expression of the strain energy of an isotropic plate is as follows

$$
U=\frac{D}{2} \int_{s}\left(\frac{\partial^{2} w}{\partial x^{2}}+\frac{\partial^{2} w}{\partial y^{2}}\right)^{2}-2(1-v)\left[\frac{\partial^{2} w}{\partial x^{2}} \frac{\partial^{2} w}{\partial y^{2}}-\left(\frac{\partial^{2} w}{\partial x \partial y}\right)^{2}\right] d s
$$

By calculating the derivatives of $w(x, y)$ we obtain

$$
\begin{array}{ll}
\frac{\partial^{2} w}{\partial x^{2}}=\cos \left(\omega_{1}\right) C_{11}^{1} \frac{\pi^{2}}{a^{2}} \sin \frac{\pi x}{a} \sin \frac{\pi y}{b} & \frac{\partial^{2} w}{\partial y^{2}}=\cos \left(\omega_{1}\right) C_{11}^{1} \frac{\pi^{2}}{b^{2}} \sin \frac{\pi x}{a} \sin \frac{\pi y}{b} \\
\frac{\partial^{2} w}{\partial y \partial x}=\cos \left(\omega_{1}\right) C_{11}^{1} \frac{\pi^{2}}{a b} \sin \frac{\pi x}{a} \sin \frac{\pi y}{b} &
\end{array}
$$

Substituting Eqs. (2.20) into (2.19) yields

$$
U=\frac{E h^{3} \pi^{4} a b}{96\left(1-v^{2}\right)} \cos \left(\omega_{1} t\right) C_{11}^{1}\left(\frac{1}{a}+\frac{1}{b}\right)^{2}
$$

\section{2. $\quad$ Modeling of the electronic card}

The simplest model for a system under a vibration excitation is given by a rigid mass supported by a linear spring and damper (Piersol and Paez, 2010). Figure 2 shows schematically a model of the electronic card which is mounted resiliently over the vibrating plate. The mass of the internal box is generally very small in comparison with the entire box (Lahdenpera, 1992).

The equation of relative motion of the system is expressed in the form

$$
Z_{1}+2 \Omega_{1} \zeta_{1} \dot{Z}_{1}+\Omega_{1}^{2} Z_{1}=-\ddot{Y}
$$

where $\Omega_{1}=\sqrt{k / m}$ is the angular frequency and $m$ and $k$ represent the moving mass and the corresponding spring stiffness, respectively. $\zeta_{1}=c / C_{c}$ is the loss factor, where $C_{c}=2 \sqrt{\mathrm{km}}=2 m \Omega_{1}$, and $c$ is the damping coefficient.

The dynamic response of the plate is expressed as follows

$$
Y(t)=w(x, y, t)=\sum_{i=1}^{N} w_{i} \cos \left(\omega_{i} t\right)
$$




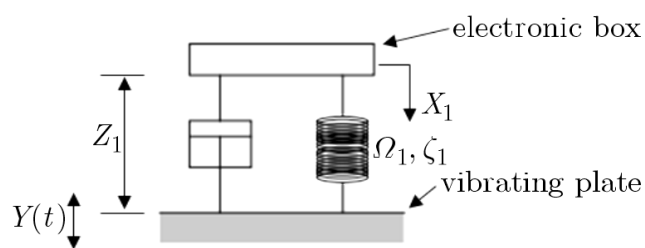

Fig. 2. Simple model of the electronic card; $\zeta_{1}$ and $\Omega_{1}$ denote, respectively, the loss factor and the natural frequency, $Y(t)$ is the plate motion, $X_{1}$ is the deflection of the system, $Z_{1}=X_{1}-Y$ denotes the relative deflection of the card to the plate

For two motors, the displacement function can be written as

$$
\begin{aligned}
& Y(t)=w(x, y, t)=w_{1} \cos \left(\omega_{1} t\right)+w_{2} \cos \left(\omega_{2} t\right) \\
& \ddot{w}(x, y, t)=-Y_{1} \cos \left(\omega_{1} t\right)-Y_{2} \cos \left(\omega_{2} t\right)
\end{aligned}
$$

where

$$
Y_{1}=\omega_{1}^{2} w_{1} \quad Y_{2}=\omega_{2}^{2} w_{2}
$$

then

$$
|Z|=\left|Z_{1}\left(\omega_{1}\right)\right|+\left|Z_{2}\left(\omega_{2}\right)\right|
$$

where

$$
\left|Z_{1}\left(\omega_{1}\right)\right|=\frac{Y_{1}}{\sqrt{\left(\Omega_{1}^{2}-\omega_{1}^{2}\right)^{2}+4 \omega_{1}^{2} \Omega_{1}^{2} \zeta^{2}}} \quad\left|Z_{2}\left(\omega_{2}\right)\right|=\frac{Y_{2}}{\sqrt{\left(\Omega_{1}^{2}-\omega_{2}^{2}\right)^{2}+4 \omega_{2}^{2} \Omega_{1}^{2} \zeta^{2}}}
$$

\section{System modeling}

Modeling language Modelica has been chosen to implement the models described above. As Modelica is an object oriented modeling language, it allows the realization of a complex system by simple gathering components (Schiavo et al., 2006). The construction of this model (Fig. 3) includes elements developed expressly for this application such as the plate component and selection of the position component as well as parts that belong to Dymola/Modelica library.

The model of the system in Dymola is decomposed into a set of connected components; everyone is represented by a block containing the necessary information about the element, which can be changed by the user. The model can be divided into five main parts. The motor represents the dynamic excitation over the exterior surface of the plate. In this case, we model the motor by a uniformly distributed $p \cos (\omega t)$-type force acting over a rectangular subdomain of the plate. To choose the location of the card on the plate, the selection of the position element has been modelled. The simply supported plate object has been modelled with consideration of the necessary equations describing vibration propagation through the structure. Consideration has been given to the principal parameters that characterize the physical properties of this plate such as the dimensions, Poisson's ratio and Young's modulus.

In Fig. 3 components of the Modelica library are used: the linear 1D model composed of a spring and damper in parallel and the sliding mass, to model the electronic card. The sensors measure absolute position, absolute velocity and absolute acceleration in order to control the displacement, speed, and acceleration of the electronic card on the plate.

To describe the physical interfaces between these components, we use connectors which are associated with every component. In order to describe the physical interfaces between the motors 


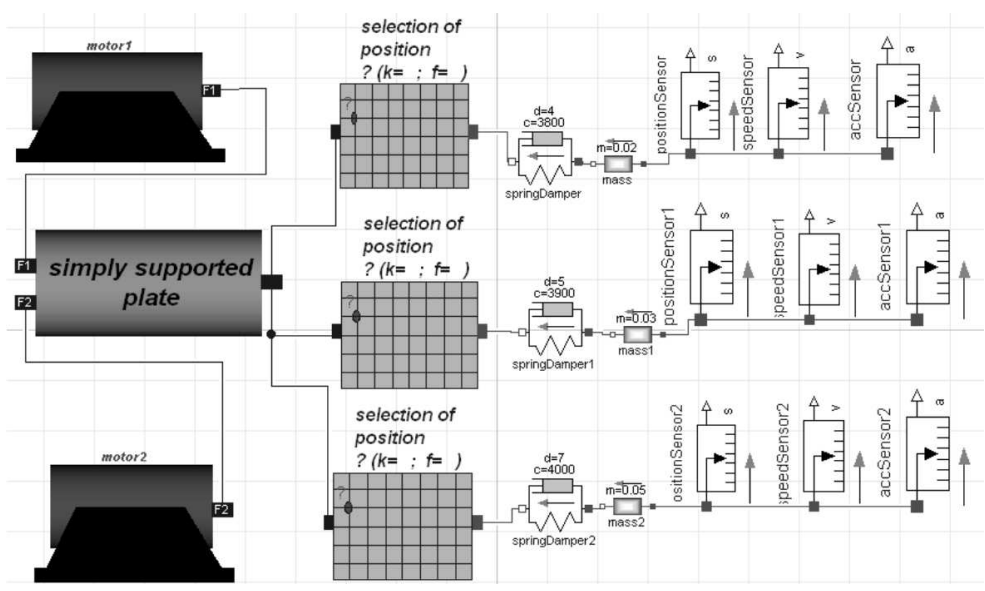

Fig. 3. Model of the system in Dymola/Modelica

Table 1. New connector properties

\begin{tabular}{|l|c|c|}
\hline \multicolumn{1}{|c|}{ Type } & Name & Description \\
\hline \hline Length & $u_{1}$ & length $[\mathrm{m}]$ \\
\hline Length & $v$ & length $[\mathrm{m}]$ \\
\hline Pressure & $P_{0}$ & force magnitude $\left[\mathrm{N} / \mathrm{m}^{2}\right]$ \\
\hline Angular velocity & $z$ & angular frequency $[\mathrm{rad} / \mathrm{s}]$ \\
\hline
\end{tabular}

and the plate, we have introduced a new connector in Dymola. Table 1 shows the characteristics of the new connector used to connect new the models build in Dymola.

This new model will give us the possibility to have a simplified procedure which will enable us to perform behaviour simulations, technology completions and to estimate the vibrational behaviour of the mechatronic system.

\section{Results and discussions}

\subsection{Case study presentation}

According to the theoretical analysis in Section 2, two motors are considered in this study and two electronic cards which are located on the rectangular plate (Fig. 4a). The finite element analysis with ANSYS (Fig. 4b) has been used to validate the model implemented in Modelica.

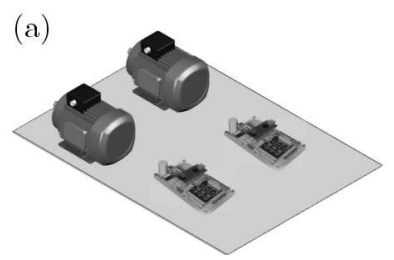

(b)

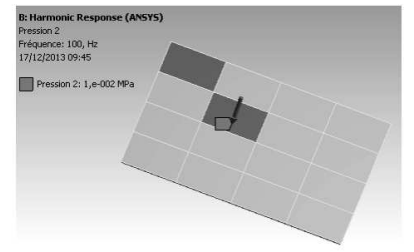

Fig. 4. (a) The studied model. (b) Modeling of the loaded plate with ANSYS

\subsection{Validation with the finite element method}

In order to validate the model implemented in Dymola/ Modelica, some results derived in this paper have been compared with those obtained with the finite element analysis using ANSYS. 
Figure $4 \mathrm{~b}$ illustrates the CAD model of the plate that has been used for this study in ANSYS WorkBench. The geometry is made of a set of rectangular faces with dimension $(u, v)$ for each face, to make easy the application of the vibrating force represented with an arrow in the figure. The mesh is not represented in this figure.

The first five natural frequencies are listed in Table 2 along with analytical results and ANSYS output data. It can be seen that the natural frequencies obtained by this method are in good agreement with those obtained by the finite element analysis using ANSYS.

Table 2. Natural frequencies $[\mathrm{Hz}]$ of the simply supported plate. $m$ and $n$ are the mode numbers in the $x$ and $y$ direction, respectively

\begin{tabular}{|c|c|c|}
\hline$(m, n)$ & Analytical solution & ANSYS \\
\hline \hline$(1,1)$ & 169.88 & 170 \\
\hline$(1,2)$ & 423.36 & 426.8 \\
\hline$(2,2)$ & 676.32 & 682.9 \\
\hline$(1,3)$ & 848.29 & 853.67 \\
\hline$(3,2)$ & 1099 & 1109 \\
\hline
\end{tabular}

Table 3 and 4 give the bending at the centre of the plate for several positions of the two motors and illustrate the error between the two methods.

Table 3. Bending at the centre of the plate, the two motors have the same amplitude of vibration, $p_{1}=p_{2}=0.01 \mathrm{MPa}$ and the same frequency, $f_{1}=f_{2}=100 \mathrm{~Hz}$

\begin{tabular}{|c|c|c|c|}
\hline Motors positions & Modelica & ANSYS & Error \\
\hline \hline $\begin{array}{c}\left(\alpha_{1}, \beta_{1}\right)=(0.03 \mathrm{~m}, 0.03 \mathrm{~m}) \\
\left(\alpha_{2}, \beta_{2}\right)=(0.03 \mathrm{~m}, 0.09 \mathrm{~m})\end{array}$ & 0.094 & 0.095 & $0.15 \%$ \\
\hline $\begin{array}{l}\left(\alpha_{1}, \beta_{1}\right)=(0.03 \mathrm{~m}, 0.03 \mathrm{~m}) \\
\left(\alpha_{2}, \beta_{2}\right)=(0.09 \mathrm{~m}, 0.09 \mathrm{~m})\end{array}$ & 0.2 & 0.2 & $0 \%$ \\
\hline $\begin{array}{l}\left(\alpha_{1}, \beta_{1}\right)=(0.03 \mathrm{~m}, 0.09 \mathrm{~m}) \\
\left(\alpha_{2}, \beta_{2}\right)=(0.09 \mathrm{~m}, 0.09 \mathrm{~m})\end{array}$ & 0.241 & 0.234 & $2.99 \%$ \\
\hline $\begin{array}{l}\left(\alpha_{1}, \beta_{1}\right)=(0.09 \mathrm{~m}, 0.09 \mathrm{~m}) \\
\left(\alpha_{2}, \beta_{2}\right)=(0.15 \mathrm{~m}, 0.15 \mathrm{~m})\end{array}$ & 0.347 & 0.359 & $3.34 \%$ \\
\hline
\end{tabular}

The results are compared with those obtained with finite element analysis using ANSYS. It can be noticed that the error between two methods is less than $6 \%$. Therefore, the results given by the analytical models and the FEM models are close to each other.

Table 4. Bending at the plate centre, the two motors have different amplitudes of vibration, $p_{1}=0.01 \mathrm{MPa}, p_{2}=0.02 \mathrm{MPa}$ and the same frequency, $f_{1}=f_{2}=100 \mathrm{~Hz}$

\begin{tabular}{|c|c|c|c|}
\hline Motors positions & Modelica & ANSYS & Error \\
\hline \hline $\begin{array}{c}\left(\alpha_{1}, \beta_{1}\right)=(0.03 \mathrm{~m}, 0.03 \mathrm{~m}) \\
\left(\alpha_{2}, \beta_{2}\right)=(0.03 \mathrm{~m}, 0.09 \mathrm{~m})\end{array}$ & 0.162 & 0.171 & $5.26 \%$ \\
\hline $\begin{array}{l}\left(\alpha_{1}, \beta_{1}\right)=(0.03 \mathrm{~m}, 0.03 \mathrm{~m}) \\
\left(\alpha_{2}, \beta_{2}\right)=(0.09 \mathrm{~m}, 0.09 \mathrm{~m})\end{array}$ & 0.374 & 0.383 & $2.34 \%$ \\
\hline $\begin{array}{c}\left(\alpha_{1}, \beta_{1}\right)=(0.03 \mathrm{~m}, 0.09 \mathrm{~m}) \\
\left(\alpha_{2}, \beta_{2}\right)=(0.09 \mathrm{~m}, 0.09 \mathrm{~m})\end{array}$ & 0.414 & 0.426 & $2.8 \%$ \\
\hline $\begin{array}{l}\left(\alpha_{1}, \beta_{1}\right)=(0.09 \mathrm{~m}, 0.09 \mathrm{~m}) \\
\left(\alpha_{2}, \beta_{2}\right)=(0.15 \mathrm{~m}, 0.15 \mathrm{~m})\end{array}$ & 0.520 & 0.541 & $3.88 \%$ \\
\hline
\end{tabular}




\subsection{Parametric study}

After the modeling of the entire system with Dymola/Modelica, an analysis has been carried out for different magnitudes. The plate is characterized by the parameters $a$ and $b$ which are lengths of the plate along, respectively, in the $x$ and $y$ direction, $a=b=240 \mathrm{~mm}$, plate thickness $h=20 \mathrm{~mm}$, density of the plate material $\rho=7850 \mathrm{Kg} / \mathrm{m}^{3}$, Poisson's ratio $v=0.3$ and Young's modulus $E=210000 \mathrm{MPa}$. Several simulations tests have been made. Figures 5a, 5b and 5c show, respectively, the displacement, speed and acceleration at the centre of the plate which is subjected to a bi-harmonic force that acts at positions $\left(\alpha_{1}, \beta_{1}\right)=(0.03 \mathrm{~m}, 0.03 \mathrm{~m})$ and $\left(\alpha_{2}, \beta_{2}\right)=$ $(0.09 \mathrm{~m}, 0.09 \mathrm{~m})$. The excitation amplitudes are $p_{1}=p_{2}=0.01 \mathrm{MPa}$ with excitation frequencies $f_{1}=f_{2}=100 \mathrm{~Hz}$.
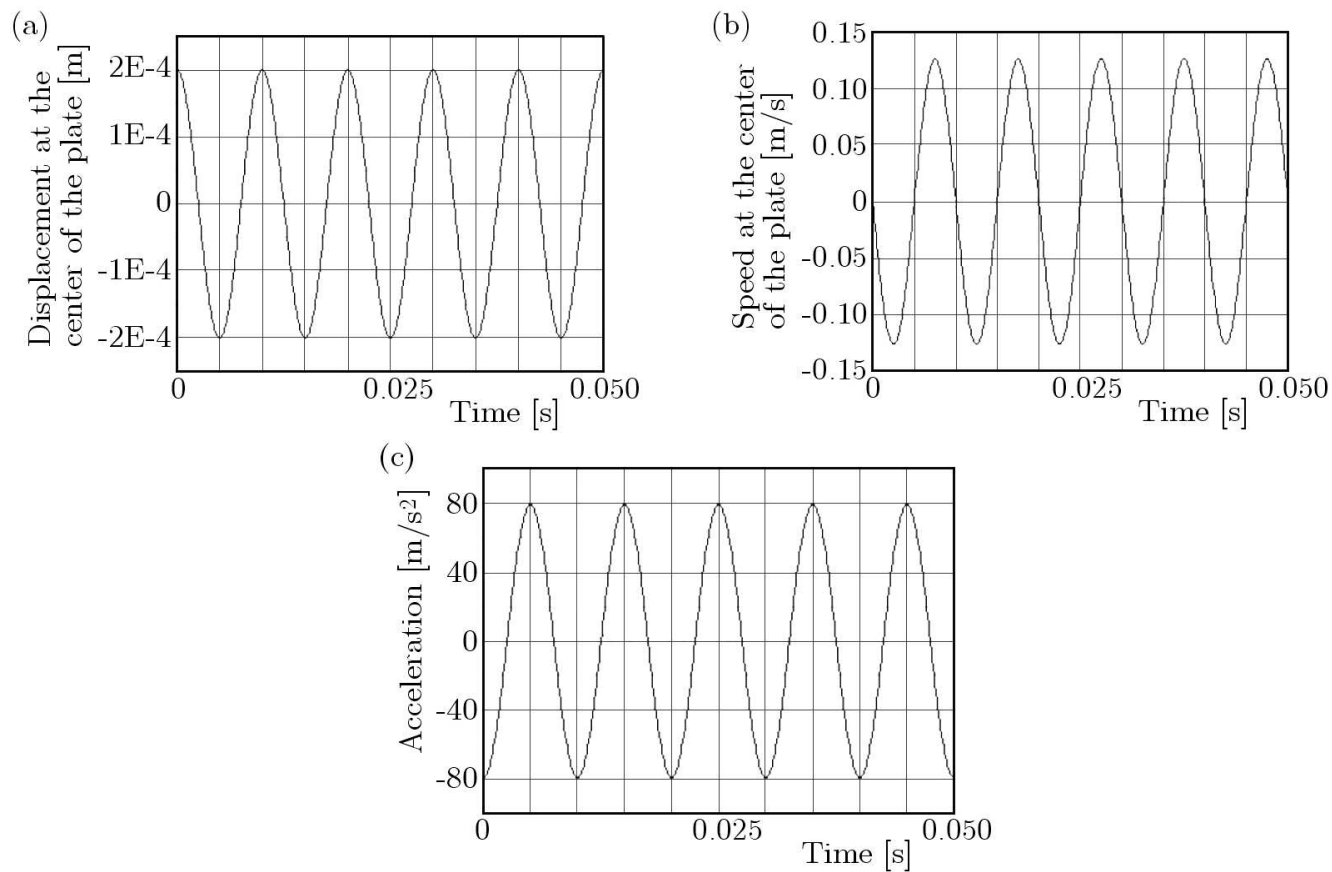

Fig. 5. Displacement (a), speed (b) and acceleration (c) at the plate centre

As it can be observed, the displacement, speed and acceleration at the centre of the plate have sinusoidal shapes with a frequency of $100 \mathrm{~Hz}$ (frequency of excitations) and they have, respectively, amplitudes of $0.2 \mathrm{~mm}, 0.125 \mathrm{~m} / \mathrm{s}$ and $79.25 \mathrm{~m} / \mathrm{s}^{2}$. The speed and the acceleration are, respectively, $\pi / 2$ and $\pi$ phase-shifted with respect to the displacement.

Comparison between the amplitudes of vibration in the centre of the rectangular plate and that of the electronic card which is located in the same position is shown in Fig. 6. From the results presented in this figure, it can be observed that the two curves are in phases. But the amplitude of vibration of the mass is lower than that of the plate centre, which is due to the damping effect. Concerning the movement of the mass, a transient phenomenon occurs at the beginning of the movement. It represents the response of the system before stabilization, which strongly depends on the initial conditions. Then, there is a steady-state regime when the initial conditions have no longer influence.

Figure 7 shows the amplitudes of vibration of some points which are located in the middle line of the plate. As it can be observed, the deflections are different from one point to another. Moreover, the period of vibration is maintained and the curves are in phases. Then for the plate excited by two excitation forces acting at different locations, regardless the parameters, we can control the vibration amplitude in any point of the rectangular plate. The curve of transverse central deflection according to the plate width is given in Fig. 8. It is shown that the amplitude of vibration increases with increasing the plate width. 


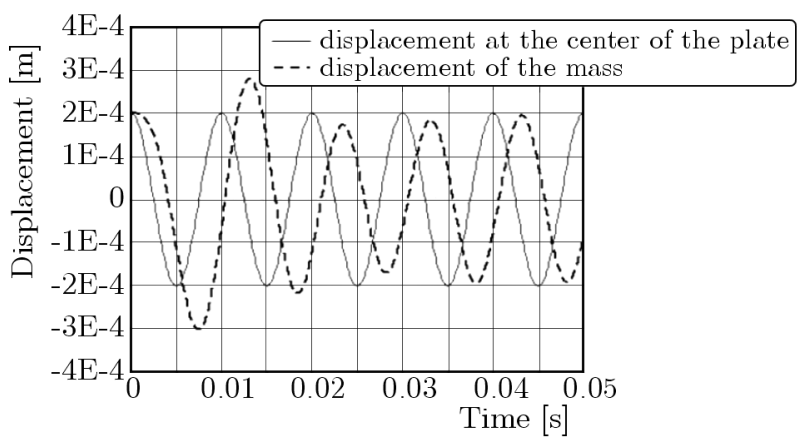

Fig. 6. Bending at the plate centre and displacement measured by the position sensor

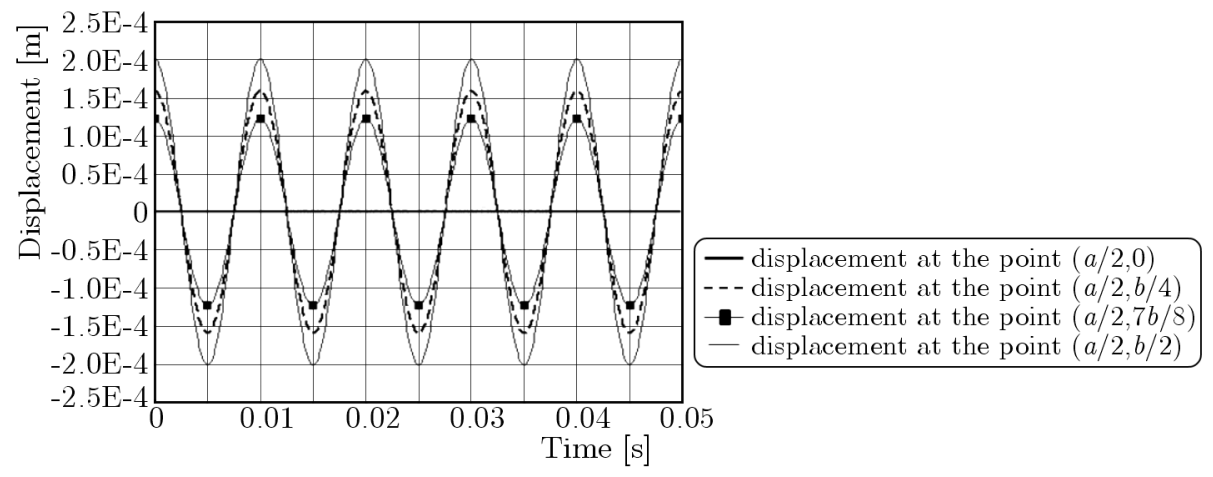

Fig. 7. Bending of the plate at different points in the middle line of the plate

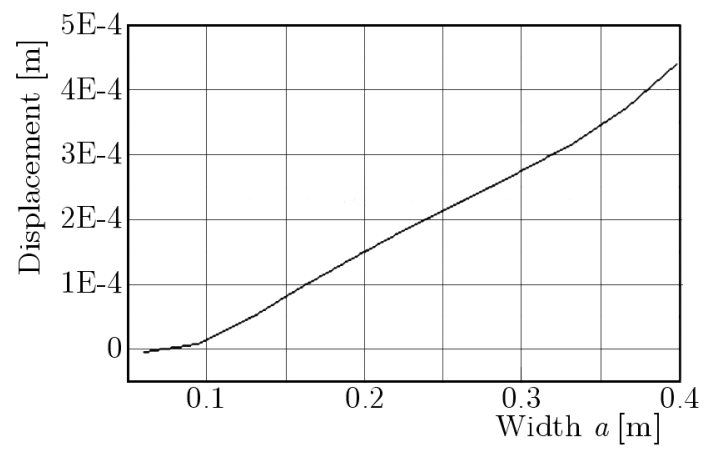

Fig. 8. Displacement at the plate centre according to its width $a$

The Navier approach is based on the thin plate theory. In this case, $h$ is assumed to be negligible relatively to other dimensions (width and length). Yet, the height value has a great impact on the vibration propagation. Figure 9a represents the central deflection according to the plate height. As illustrated, the curve of the height has two parts. In the beginning, the vibration amplitude increases with the increasing height of the plate until reaching the value of $0.0029 \mathrm{~m}$, then there is a decrease in the amplitude when the height increases, the amplitude of vibration tends to zero. Deflection of the plate depends mainly on its dimensions. Figure $9 \mathrm{~b}$ clearly shows the variation of transverse displacement at the centre of the plate according to its dimensions $(a, b)$. So, it can be seen that as the dimension values increase, the vibration amplitude increases.

Figures 10a and 10b depict, respectively, the central deflection of the plate according to the first motor dimensions and the motor positions on the rectangular plate while keeping other parameters fixed. The influence of the angular frequency on the vibrational response of the plate is given in Fig. 10c. 

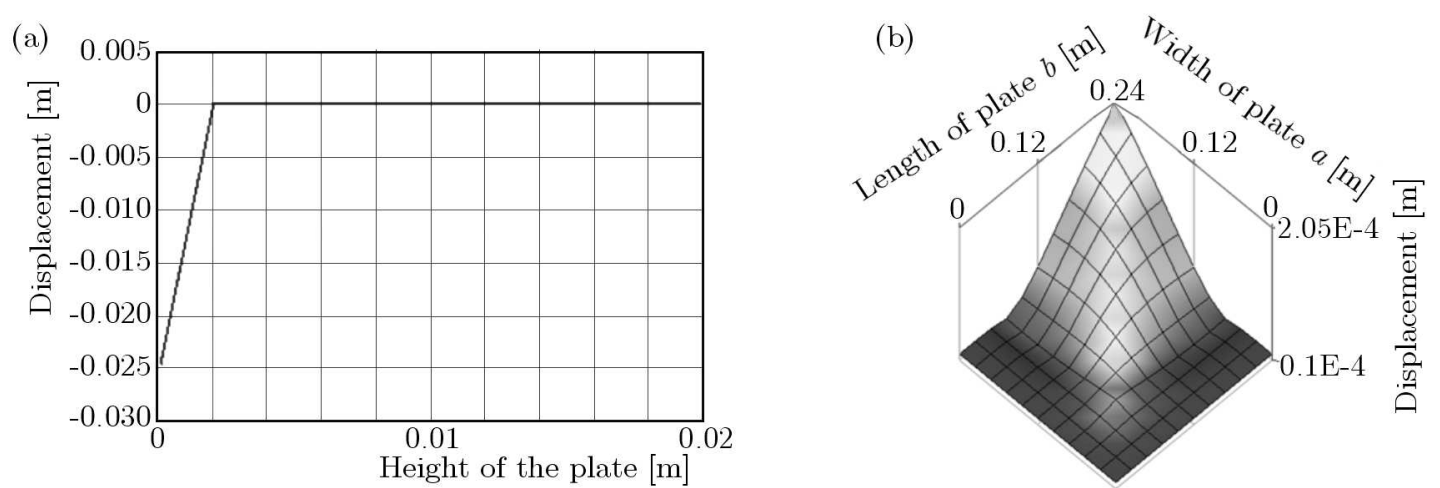

Fig. 9. Displacement at the plate centre according to: (a) its height $h$, (b) plate dimensions

(a)

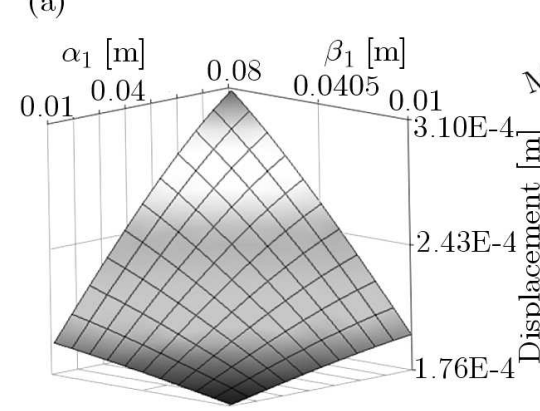

(b)

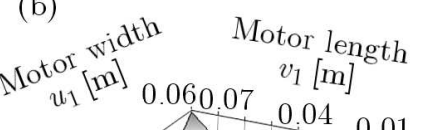

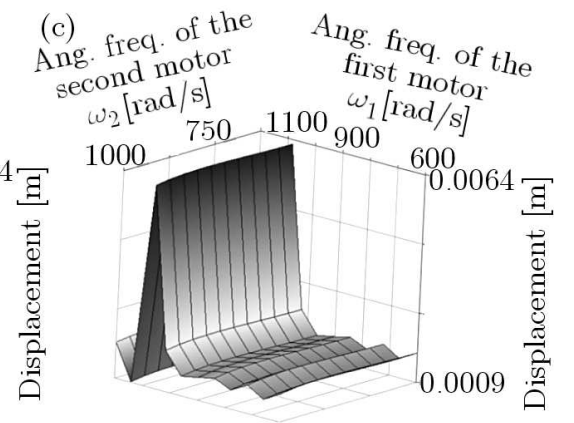

Fig. 10. Displacement at the plate centre according to: (a) dimensions of the first motor $\left(\alpha_{1}, \beta_{1}\right)$, (b) the motor positions $\left(u_{1}, v_{1}\right)$, (c) the angular frequencies of the motors

Figures 11a and 11b show the effect of dimensions variation (width and length) on the strain and kinetic energy of the simply supported rectangular plate with uniform thickness. It can be observed that the kinetic energy increases with an increasing in the width $a$, in addition, the plate dimensions have a significant impact on the plate energies. At any position of the electronic card over the rectangular plate, we can control its motion.
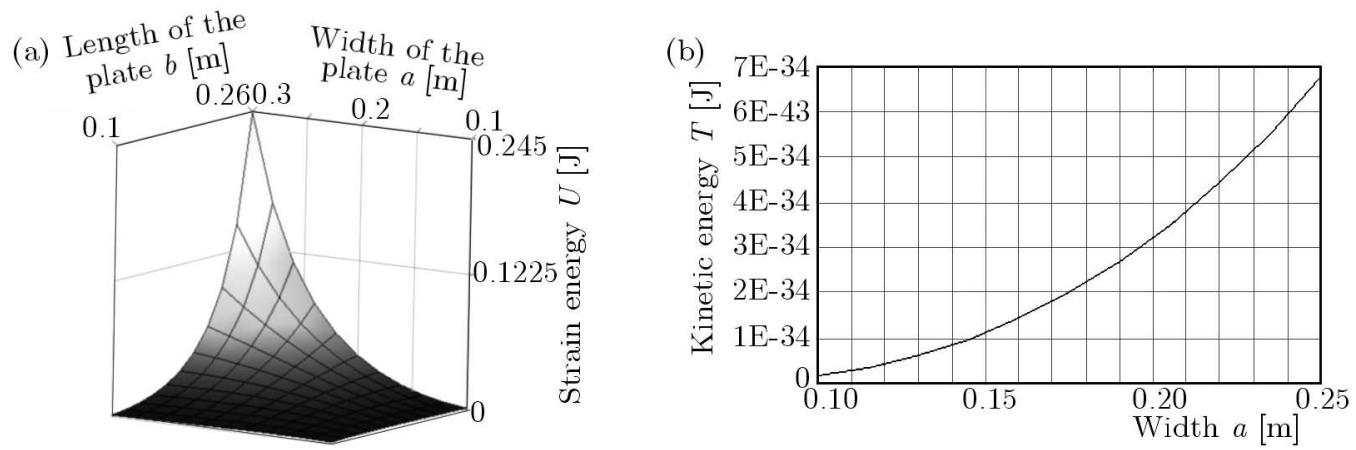

Fig. 11. (a) Strain energy of the plate according to its dimensions $(a, b), p_{1}=0.01 \mathrm{MPa}$, $\left(\alpha_{1}, \beta_{1}\right)=(0.03 \mathrm{~m}, 0.03 \mathrm{~m})$. (b) Kinetic energy of the plate according to its width $a$

The simulation results of the speed and acceleration control of the electronic card are given in Figs. 12a and 12b. The card is located in the centre of the plate. As it is shown, the speed and acceleration are sinusoidal functions; both curves show a transient regime in the beginning, and then a steady-state regime is established. In addition, the two amplitudes depend strongly on the card characteristics as well as the card position on the plate. 

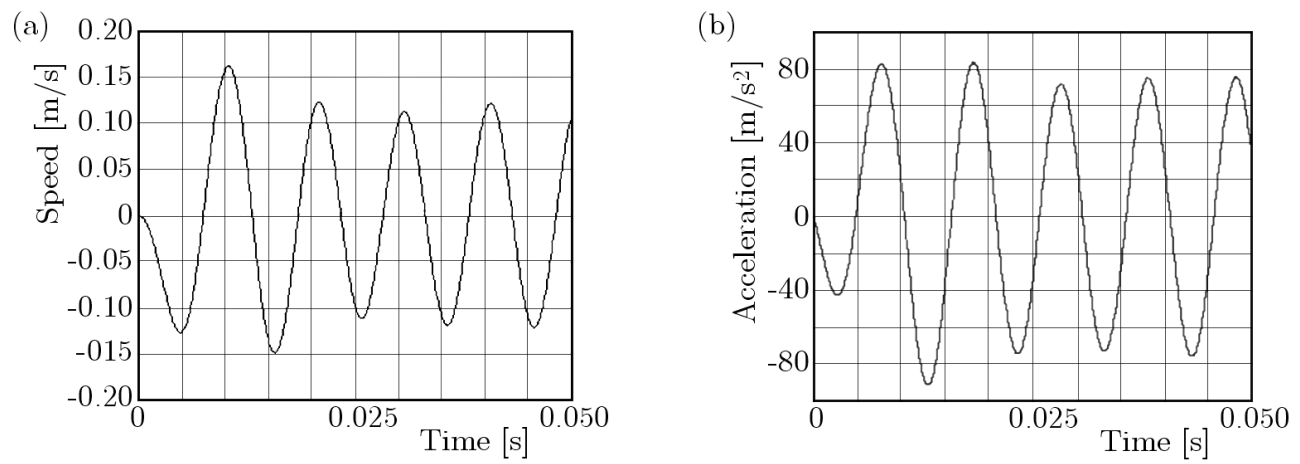

Fig. 12. (a) Speed measured by the speed sensor. (b) Acceleration response of the mass

\section{Conclusions}

In this paper, we have proposed a new approach for the preliminary design of a mechatronic system based on an analytical method. We defined a model that allows the predictability of the vibrational behaviour of the system. The model has been developed using the modeling language Modelica/Dymola. To show the capability of the model to help designers in pre-dimensioning, we have analysed several parameters having significant influence on the vibration propagation. The possibility of modifing the geometrical characteristic, the parameters and the evaluation of possible missions will serve as a strong positive point for this new method. This modeling process will serve to meet the system requirements with acceptable risks and to establish the basis for proceeding with a detailed design. The simulation results have been validated with the finite element analysis using ANSYS.

Only harmonic excitation has been considered in this study. This work will be extended to include the optimization of mechatronic systems under stochastic vibration.

\section{References}

1. Bhaskar K., Sivaram A., 2008, Untruncated infinite series superposition method for accurate flexural analysis of isotropic/orthotropic rectangular plates with arbitrary edge conditions, Composite Structures, 8, 83-92

2. Craig K., Stolfi F., 2002, Teaching control system design through mechatronics: academic and industrial perspectives, Mechatronics, 12, 371-381

3. Elmqvist H., Mattsson S.E., Otter M., 2001, Object-oriented and hybrid modeling in Modelica, Journal Européen des Systèmes Automatisés, 35, 1-22

4. Hammadi M., Choley J.Y., Penas O., Rivère A., 2012a, Multidisciplinary approach for modelling and optimization of road electric vehicles in conceptual design level, Electrical Systems for Aircraft, Railway and Ship Propulsion (ESARS), IEEE, 1-6

5. Hammadi M., Choley J.Y., Penas O., Rivère A., Louati J., Haddar M., 2012b, A new multi-criteria indicator for mechatronic system performance evaluation in preliminary design level, 13th International Workshop on Mechatonics, 9th France-Japan and 7th Europe-Asia Congress on Research and Education in Mechatronics (REM), 409-416

6. Lahdenpera M., 1992, Severe environment electronics, Mechatronics, 2, 301-309

7. Laura P.A.A., Duran R., 1975, A note on forced vibrations of a clamped rectangular plate, Journal of Sound and Vibration, 42, 129-135

8. Leissa A.W., 2005, The historical bases of the Rayleigh and Ritz method, Journal of Sound and Vibration, 287, 961-978 
9. Liscouët J., Maré J.C., Budinger M., 2012, An integrated methodology for the preliminary design of highly reliable electromechanical actuators: search for architecture solutions, Aerospace Science and Technology, 22, 9-18

10. Mishra S., Pecht M., 2002, Remaining life prediction of electronic products using life consumption monitoring approach, European Microelectronics Packaging and Interconnection Symposuim, Cracow, Poland, 16-18 June.

11. Piersol A.G., Paez T.L., 2010, Harris' Shock and Vibration Handbook, 6th edition, United States

12. Romanelli E., Laura P.A.A., 2001, Forced transverse vibrations of a simply supported rectangular orthotropic plate in the case where the force acts over a plate subdomain, Ocean Engineering, 28, 1135-1144

13. Schiavo F., Vigano L., Ferretti G., 2006, Object-oriented modeling of flexible beams, Multibody System Dynamics, 15, 3, 263-286

14. Schwartz L., 1951, Theory of Distributions (in French), tome 2, Paris, Hermann

15. SzIlard R., 2004, Theories and Applications of Plate Analysis: Classical, Numerical and Engineering Methods, John Wiley \& Sons, Inc., New Jersey

16. Tarnowski W., Krzyzynski T., Maciejewski I., Oleskiewicz R., 2011, Poly-optimization: a paradigm in engineering design in mechatronics, Archive of Applied Mechanics, 81, 141-156

17. Ventsel E., Krauthammer T., 2001, Thin Plates and Shells, Theory, Analysis, and Applications, Basel, New York

18. Veprik A.M., BABtisky V.I., 2000, Vibration protection of sensitive electronic equipment from harsh harmonic vibration, Journal of Sound and Vibration, 238, 19-30

19. Vera S.A., Febbo M., Mendez C.G., Paz R., 2005, Vibrations of a plate with an attached two degree of freedom system, Journal of Sound and Vibration, 285, 457-466

20. Wang Y., Yu Y., Xie C., Zhang X., Jiang W., 2013, A proposed approach to mechatronics design education: integrating design methodology, simulation with projects, Mechatronics, 23, 942-948

21. Wei G.W., Zhao Y.B., Xiang Y., 2001, The determination of natural frequencies of rectangular plates with mixed boundary conditions by discrete singular convolution, International Journal of Mechanical Sciences, 43, 1731-1746

22. Wu J.S., LuO S.S., 1997, Use of the analytical and numerical combined method in the free vibration analysis of a rectangular plate with any number of point mass and translational springs, Journal of Sound and Vibration, 200, 179-194 